\title{
Square cells in gravitational and capillary thermoconvection
}

\author{
V. Regnier,* P. C. Dauby, P. Parmentier, and G. Lebon ${ }^{\dagger}$ \\ Université de Liège, Institut de Physique B5, Sart Tilman, B 4000 Liège 1, Belgium
}

(Received 4 December 1996)

\begin{abstract}
The onset of square convective cells in fluid layers heated from below is investigated. Amplitude equations are deduced from the Boussinesq equations and a standard stability analysis is performed. Square cells are shown to be preferred when the instability is mainly capillarity driven. The influence of the Prandtl and Biot numbers are examined. At small Pr, the Biot number has not very much influence and squares are always observed for thin enough layers. In large Prandtl number fluids, Bi must be larger than the limiting value 0.28 for squares to be stable. [S1063-651X(97)10405-6]
\end{abstract}

PACS number(s): 47.20.-k, 44.25.+f, 47.27.Te

\section{INTRODUCTION}

Pattern formation in thermoconvection has been the object of a large amount of interest for many years and many scientists have analyzed this problem. When a horizontal fluid layer is heated from below, convection sets in after a critical temperature difference between the bottom and the top of the liquid has been reached. The motion that appears above the threshold is generally well structured and a regular pattern of convective cells may be observed.

The geometrical nature of the convective cells that appear above the threshold depends greatly on the mechanism that causes the instability: usually, rolls are observed in gravitydriven convection while hexagonal cells are preferred when the motion originates in capillary effects. We will not review here all the papers on this subject $[1-20]$ since the main results were commented on by Parmentier, Regnier, Lebon, and Legros [21].

Very recently, Nitschke and Thess [22] reported experiments in which square convective cells were observed in surface-tension-driven convection at a relative distance from threshold larger than about 2.35. A fast theoretical interpretation was proposed by Bestehorn [23] for pure capillary (or Marangoni) convection. Its study is based on direct numerical simulation of the Navier-Stokes equations as well as on amplitude equations deduced from a model equation for convection. Recently, Bragard [24] deduced amplitude equations from the complete field equations in the case of pure Marangoni instability and he showed that square patterns are theoretically observable only if the Biot number at the upper free surface is nonzero. Another recent paper by Golovin, Nepomnyashchy, and Pismen [25] also considers the problem of square convective cells in a two layer liquid-gas system with a deformable interface but, like all the previous authors, they neglect gravity. The competition between square planforms and hexagonal structures was also studied from a theoretical point of view by Kubstrup et al. [26] who used generalized Swift-Hohenberg equations.

The purpose of the present paper is to examine the

\footnotetext{
*Electronic address: vregnier@ulg.ac.be

${ }^{\dagger}$ Also at Louvain University, Department of Mechanics, B 1348 Louvain-La-Neuve, Belgium.
}

coupled gravity- and capillarity-driven instability, with special emphasis on the possibility of the occurrence of square structures. Clearly, this model is better suited to interpret the experiments of Nitschke and Thess, which were realized on Earth. From a technical point of view, our approach complements our previous analysis of rolls and hexagonal cells [21]. In Sec. II, the Landau amplitude equations for the roll mode, the square structure, and the hexagonal cells is derived. In the next section, the stability of the solutions corresponding to the different planforms will be examined while the influence of the Prandtl and Biot numbers is studied in Sec. IV. Final conclusions are drawn in the last section.

\section{LANDAU EQUATIONS FOR ROLLS, HEXAGONAL CELLS, AND SQUARE CONVECTIVE PATTERNS}

The procedure followed here to obtain the amplitude equations for the roll, hexagonal, and square structures is similar to that used in our previous work [21]. For this reason, most of the technical details will be omitted in this work; moreover, when notation is the same, it will not always be redefined.

The linear study of stability is not modified with respect to the work of Parmentier et al. [21] and will not be commented upon any further here. The nonlinear approach to the problem is based on the development of the solution in eigenmode series of the linear problem (considered as an eigenvalue problem for the growth rate $s$ with the Marangoni and Rayleigh numbers fixed at their critical values). With horizontal dependence of the form $\exp \left[i\left(k_{x} x+k_{y} y\right)\right]$ for the eigenmodes, the development is written as

$$
f=\sum_{p=0}^{N_{p}-1} \sum_{k} A_{s_{p}}^{\mathbf{k}} f_{s_{p}}^{\mathbf{k}}, \quad \mathbf{k} \in K,
$$

where $f$ denotes the unknown fields and $s_{p}$ the successive eigenvalues. In order that $f$ be real, the amplitudes must satisfy

$$
\overline{A_{s_{p}}^{\mathbf{k}}}=A \frac{-\mathbf{k}}{s_{p}},
$$

where the overbar denotes the complex conjugate.

The set $K$ is made up of $K_{c}$ and $K_{s}$, which contain, respectively, the critical eigenmodes taken into account and the 


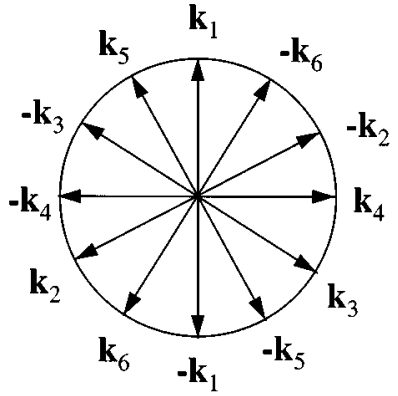

FIG. 1. Wave vectors for rolls, hexagons, and squares.

modes generated by the quadratic interactions of the elements of $K_{c}$. To describe the interactions of rolls, square structure, and hexagonal cells, the set $K_{c}$ consists of the 12 vectors $\pm \mathbf{k}_{i}(i=1, \ldots, 6)$ with an angle of $30^{\circ}$ between them and represented in Fig. 1. The corresponding elementary geometrical structures are 6 rolls with maximal vertical velocity at the origin making angles of $30^{\circ}$ and 6 other rolls, which are spatially out of phase by $\pi / 2$ (i.e., that are moved normally to themselves by half a roll width).

When development (1) is introduced in the Boussinesq balance equations, the following Landau equations are obtained for the six complex amplitudes $A_{i}=A_{s_{0}}^{\mathbf{k}_{i}}$ (with $s_{0}=0$ and $i=1, \ldots, 6)$ :

$$
\begin{aligned}
\tau \frac{d A_{1}}{d t}= & \epsilon A_{1}+a \overline{A_{2}} \overline{A_{3}}-b\left(\left|A_{2}\right|^{2}+\left|A_{3}\right|^{2}\right) A_{1}-c\left|A_{1}\right|^{2} A_{1} \\
& -d\left(\left|A_{5}\right|^{2}+\left|A_{6}\right|^{2}\right) A_{1}-e\left|A_{4}\right|^{2} A_{1}, \\
\tau \frac{d A_{2}}{d t}= & \epsilon A_{2}+a \overline{A_{3}} \overline{A_{1}}-b\left(\left|A_{3}\right|^{2}+\left|A_{1}\right|^{2}\right) A_{2}-c\left|A_{2}\right|^{2} A_{2} \\
& -d\left(\left|A_{6}\right|^{2}+\left|A_{4}\right|^{2}\right) A_{2}-e\left|A_{5}\right|^{2} A_{2}, \\
\tau \frac{d A_{3}}{d t}= & \epsilon A_{3}+a \overline{A_{1}} \overline{A_{2}}-b\left(\left|A_{1}\right|^{2}+\left|A_{2}\right|^{2}\right) A_{3}-c\left|A_{3}\right|^{2} A_{3} \\
& -d\left(\left|A_{4}\right|^{2}+\left|A_{5}\right|^{2}\right) A_{3}-e\left|A_{6}\right|^{2} A_{3},
\end{aligned}
$$

$$
\begin{aligned}
\tau \frac{d A_{4}}{d t}= & \epsilon A_{4}+a \overline{A_{5}} \overline{A_{6}}-b\left(\left|A_{5}\right|^{2}+\left|A_{6}\right|^{2}\right) A_{4}-c\left|A_{4}\right|^{2} A_{4} \\
& -d\left(\left|A_{2}\right|^{2}+\left|A_{3}\right|^{2}\right) A_{4}-e\left|A_{1}\right|^{2} A_{4},
\end{aligned}
$$

$$
\begin{aligned}
\tau \frac{d A_{5}}{d t}= & \epsilon A_{5}+a \overline{A_{6}} \overline{A_{4}}-b\left(\left|A_{6}\right|^{2}+\left|A_{4}\right|^{2}\right) A_{5}-c\left|A_{5}\right|^{2} A_{5} \\
& -d\left(\left|A_{3}\right|^{2}+\left|A_{1}\right|^{2}\right) A_{5}-e\left|A_{2}\right|^{2} A_{5},
\end{aligned}
$$

$$
\begin{aligned}
\tau \frac{d A_{6}}{d t}= & \epsilon A_{6}+a \overline{A_{4}} \overline{A_{5}}-b\left(\left|A_{4}\right|^{2}+\left|A_{5}\right|^{2}\right) A_{6}-c\left|A_{6}\right|^{2} A_{6} \\
& -d\left(\left|A_{1}\right|^{2}+\left|A_{2}\right|^{2}\right) A_{6}-e\left|A_{3}\right|^{2} A_{6},
\end{aligned}
$$

where $\epsilon=\left(\mathrm{Ra}-\mathrm{Ra}_{c}\right) / \mathrm{Ra}_{c}=\left(\mathrm{Ma}-\mathrm{Ma}_{c}\right) / \mathrm{Ma}_{c}$ is the relative distance to the threshold ( $\mathrm{Ra}$ and $\mathrm{Ma}$ are the Rayleigh and
Marangoni numbers defined, for instance, in [21]; $\mathrm{Ra}_{c}$ and $\mathrm{Ma}_{c}$ are the corresponding critical values that define the linear instability threshold).

The equations for $A_{-i}=A_{\bar{s}_{0}}^{-\mathbf{k}_{i}}$ (with $S_{0}=0$ ) are the complex conjugates of the preceding set and are thus equivalent to it.

Apart from the trivial conductive solution $\left(A_{i}=0\right)$, the solutions corresponding to the different symmetries are

$$
A_{1}=A_{R}, \quad A_{j \neq 1}=0 \quad \text { (rolls), }
$$

$$
A_{1}=A_{2}=A_{3}=A_{H}^{ \pm}, \quad A_{4}=A_{5}=A_{6}=0 \quad \text { (hexagons), }
$$

$$
A_{1}=A_{4}=A_{S}, \quad A_{j}=0(1 \neq j \neq 4) \quad \text { (squares). }
$$

In these expressions, the amplitudes $A_{R}, A_{H}$, and $A_{S}$ for rolls, hexagons, and squares, repectively, are given by

$$
\begin{gathered}
A_{R}^{2}=\frac{\epsilon}{c}, \\
A_{H}^{ \pm}=\frac{a \pm \sqrt{a^{2}+4 \epsilon(c+2 b)}}{2(c+2 b)}, \\
A_{S}^{2}=\frac{\epsilon}{c+e} .
\end{gathered}
$$

Note that (7) and (9) imply that supercritical rolls or squares can be found only if $c>0$ or $c+e>e$, respectively. In the expression (8) for the amplitude of the hexagonal cells, the signs " " " or " - " can be seen to correspond, respectively, to upflow or downflow at the center of the cells for positive $a$ 's or the opposite for negative $a$ 's. It is worth noticing that other solutions with the same symmetries are possible; these can be obtained by rotating or translating the solutions given by Eqs. (4-6) and are thus equivalent to them.

\section{STABILITY ANALYSIS}

To examine the stability of the different solutions, the system (3) is first transformed into a set of 12 real equations by taking the real and imaginary parts of each equation. Then a standard linear perturbation analysis is performed for each pattern and 12 eigenvalues $\sigma_{i}$ are determined, which must be negative for stability. It is interesting to examine in detail the eigenvalues in the different structures.

For the roll pattern, the eigenvalues are given by

$$
\begin{gathered}
\sigma_{1,2}=\epsilon+a A_{R}-b A_{R}^{2}, \quad \sigma_{3,4}=\epsilon-a A_{R}-b A_{R}^{2}, \\
\sigma_{5}=0, \quad \sigma_{6}=-2 \epsilon \\
\sigma_{7-10}=\epsilon\left(1-\frac{d}{c}\right), \quad \sigma_{11,12}=\epsilon\left(1-\frac{e}{c}\right) .
\end{gathered}
$$

The eigenvectors corresponding to the first four eigenvalues define rhomboids whose borders are at $60^{\circ}$ with the rolls. Expressions (10) show that these rhombs destabilize the roll pattern for $\epsilon<\epsilon_{R}=c a^{2} /(b-c)^{2}$. The zero value of $\sigma_{5}$ means that the pattern is indifferent to a translation perpen- 
dicular to the rolls. The eigenvalue $\sigma_{6}$ corresponds to a perturbation by the roll itself and its value shows that no subcritical convection is possible. The planforms for $\sigma_{7-10}$ are rolls at $30^{\circ}$ or $150^{\circ}$ while the eigenvectors for $\sigma_{11,12}$ are rolls at $90^{\circ}$; it is also interesting to notice that, above the threshold the rolls at $90^{\circ}$ are always destabilizing when $c>e$.

The $\sigma_{i}$ for the hexagonal cells are written as

$$
\begin{gathered}
\sigma_{1,2}=0, \quad \sigma_{3}=-3 a A_{H}^{ \pm}, \\
\sigma_{4,5}=\epsilon-a A_{H}^{ \pm}-3 c A_{H}^{ \pm^{2}}, \\
\sigma_{6}=-2 \epsilon-a A_{H}^{ \pm}, \\
\sigma_{7-12}=\epsilon-(2 d+e) A_{H}^{ \pm^{2}} .
\end{gathered}
$$

The $\sigma_{1}$ and $\sigma_{2}$ zero eigenvalues are a consequence of the translation invariance and the corresponding eigenvectors are rhomboids made up by 2 rolls parallel to the sides of the hexagons but out of phase by half a roll width with respect to the rolls constituting the hexagons. The third eigenvalue is always negative for a stable hexagonal pattern, which means that the corresponding pattern is never destabilizing for the hexagons. Note that this pattern consists of a lattice of equilateral triangles with alternatively upwards and downwards motion; the corresponding convective cells consist of some kind of hexagonal cells. The eigenvectors for $\sigma_{4,5}$ are rhomboids made up of two constitutive rolls of the hexagons with amplitudes of opposite sign. They are destabilizing for $\epsilon$ $>\epsilon_{H_{1}}=a^{2}(b+2 c) /(b-c)^{2}$. The value of $\sigma_{6}$ allows one to determine the limit $\epsilon_{c}=-a^{2} / 4(2 b+c)$ of the subcritical domain, which is defined as the $\epsilon$ interval $\left[\epsilon_{c}, 0\right]$ in which hexagonal cells may appear under the linear threshold. The last eigenvalues $\sigma_{7-12}$ correspond to rolls with axes at $90^{\circ}$ with the borders of the hexagons. These rolls are destabilizing for $\epsilon>\epsilon_{\mathrm{H}_{2}}=-a^{2}(2 d+e) /(2 d+e-2 b-c)^{2}$. We will use the symbol $\epsilon_{H}$ for the minimum value of $\epsilon_{H_{1}}$ and $\epsilon_{H_{2}}$ so that hexagons are always unstable for $\epsilon>\epsilon_{H}$.

For the square solution, the eigenvalues are given by

$$
\begin{gathered}
\sigma_{1-4}=\epsilon+a A_{c}-(b+d) A_{c}^{2}, \quad \sigma_{5-8}=\epsilon-a A_{c}-(b+d) A_{c}^{2}, \\
\sigma_{9}=2 \epsilon(e-c) /(e+c), \quad \sigma_{10}=-2 \epsilon \\
\sigma_{11,12}=0 .
\end{gathered}
$$

The first 8 eigenvalues correspond to rhomboids with sides not parallel to the sides of the square. Expressions (17) show that these rhomboids destabilize the square pattern for $\epsilon$ $<\epsilon_{S}=a^{2}(c+e) /(b+d-c-e)^{2}$. The perturbation corresponding to $\sigma_{9}$ is a square, which is spatially out of phase by half a square width; moreover, it follows from expression $\sigma_{9}=2 \epsilon(e-c) /(e+c)$ that supercritical squares are unstable for $e>c$. As it was shown previously that supercritical rolls are unstable for $c>e$, it is deduced that squares and rolls are never simultaneously stable. The negative value of $\sigma_{10}$ indicates that no subcritical squares can be observed; the eigenvector is the square itself. Finally, the two zero eigenvalues $\sigma_{11,12}$ originate in the translation invariance; the eigenvectors are the rolls constituting the square cells.
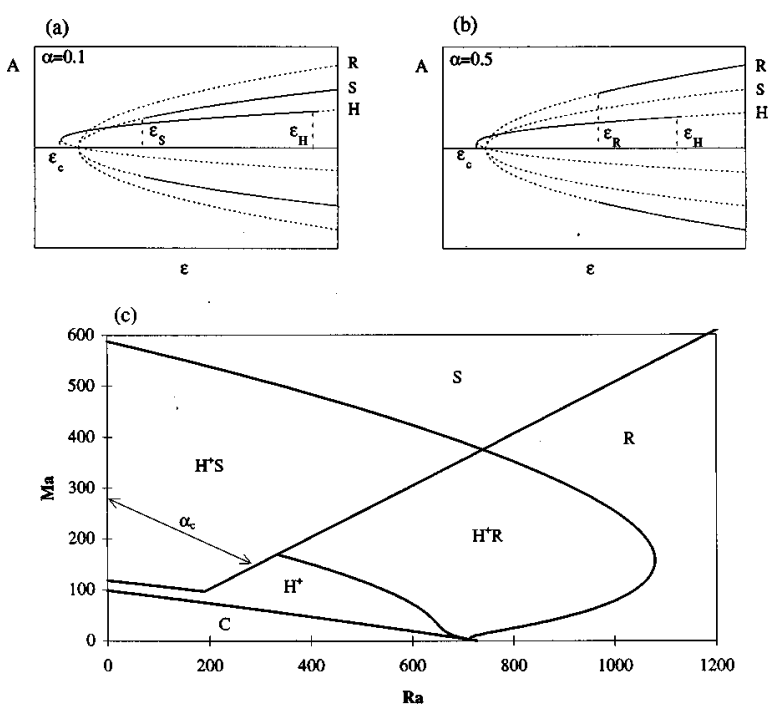

FIG. 2. Results of the stability analysis for $\operatorname{Pr}=100, \mathrm{Bi}=0.5$; (a) and (b) are the bifurcation diagrams corresponding to $\alpha=0.1$ and $\alpha=0.5$, respectively; solid and dashed lines characterize stable and unstable solutions; (c) gives the stable patterns in the Ra-Ma plane $\left(C, H^{+}, H^{-}, R\right.$, and $S$ mean conductive solution, upflowing hexagons, downflowing hexagons, rolls, and squares, respectively).

As an example, the results of the stability analysis for $\operatorname{Pr}=100, \mathrm{Bi}=0.5$ are presented in Fig. 2. For completeness, let us recall the definition of the parameters $\alpha$ and $\lambda$ in terms of the Rayleigh and Marangoni numbers:

$$
\mathrm{Ra}=\mathrm{Ra}_{0} \alpha \lambda, \quad M a=\operatorname{Ma}_{0}(1-\alpha) \lambda,
$$

where $\mathrm{Ra}_{0}$ and $\mathrm{Ma}_{0}$ are two arbitrary constants. It is easy to show that $\lambda$ is proportional to the temperature difference $\left[\lambda=\mu^{-1} \kappa^{-1}\left(\gamma \mathrm{Ma}_{0}^{-1} d+\rho g \alpha_{T} \mathrm{Ra}_{0}^{-1} d^{3}\right) \Delta T\right.$; see notation in [21]] while $\alpha$ depends on the fluid properties and the depth of the layer $\left[\alpha=\left(1+\mathrm{Ra}_{0} \gamma / \mathrm{Ma}_{0} g \rho \alpha_{T} d^{2}\right)^{-1}\right]$. Figures 2(a) and 2(b) show two bifurcation diagrams corresponding to a thin $(\alpha=0.1)$ and a thicker $(\alpha=0.5)$ fluid layer, respectively. The values of the coefficients of the corresponding Landau equations are given in Table I. In Fig. 2(a), we observe that rolls are never stable because $e>c$. It is also worth

TABLE I. The critical wave number, Marangoni and Rayleigh numbers as well as the Landau coefficients for Eq. (3) are given for two values of $\alpha$. The Prandtl and Biot numbers are given by $\operatorname{Pr}$ $=100, \mathrm{Bi}=0.5$. The normalization condition for the linear eigenmodes is a temperature equal to one at the upper free surface.

\begin{tabular}{lcc}
\hline \hline & $\alpha=0.1$ & $\alpha=0.5$ \\
\hline$k_{c}$ & 2.1379 & 2.1476 \\
$\mathrm{Ma}_{c}$ & 88.060 & 47.148 \\
$\mathrm{Ra}_{c}$ & 82.226 & 396.22 \\
$\tau$ & 0.13510 & 0.13032 \\
$a$ & 1.9790 & 1.0313 \\
$b$ & 44.999 & 31.835 \\
$c$ & 34.852 & 24.705 \\
$d$ & 56.686 & 42.679 \\
$e$ & 31.832 & 31.442 \\
\hline \hline
\end{tabular}


stressing that a hysteresis loop appears between hexagonal convection and a square pattern. In Fig. 2(b), squares are never stable and rolls appear for $\epsilon>\epsilon_{R}$. In both cases, the stable hexagonal patterns are characterized by upflow in the center of the cells because coefficient $a$ is positive. Figure 2 (c) is a summary of the results for $\operatorname{Pr}=100, \mathrm{Bi}=0.5$, whatever the value of the thickness of the layer, that is, whatever the value of $\alpha$. We have drawn in the Ra-Ma plane the different areas corresponding to the different stable convective patterns. Recall that in such a picture, a progressive heating is described by a motion along a straight line passing through the origin. Moreover, small thicknesses (i.e., small $\alpha$ ) are represented by small angles between the straight line and the vertical Ma axis while thicker layers correspond to more horizontal heating lines. In this figure and in the following, $C, H^{+}, H^{-}, R$, and $S$ indicate the stable areas for conductive solution, upflow or downflow hexagons, rolls, and squares, respectively. The following comments can be made about Fig. 2(c). One of the most important results is that square structures will not be observed in thick fluid layers while these may appear in thin layers with small values of $\alpha$. In contrast, rolls are observable only for rather large thicknesses. The border separating the roll and square regions is a straight line passing through the origin and that corresponds to a critical value $\alpha_{c}$, that is a critical thickness of the fluid layer. This $\alpha_{c}$ value is defined by the equality of the Landau coefficients $e$ and $c$. It is also seen that stable squares are merging at a distance from threshold much smaller than that for rolls: indeed the pure $\mathrm{H}^{+}$area is quite thinner for small $\alpha$ than for large $\alpha$. Note eventually that the subcritical hexagonal convection domain cannot be seen on the picture owing to its smallness.

\section{INFLUENCE OF THE PRANDTL AND BIOT NUMBERS}

In this section, we would like to discuss further the results when the Prandtl or the Biot number is changed. Rather than drawing diagrams in the Ra-Ma plane, we present the results as functions of $\alpha$. We consider first different values of Bi for $\operatorname{Pr}=100$. This Prandtl number can be considered as a "large" Prandtl number, which is typical of silicone oils used in many experiments. In particular, the fluid used in the experiments of Nitschke and Thess [22] was characterized by $\operatorname{Pr}=100$. The main results are represented in Figs. 3(a) $-3(b)$. Recall first that only upflowing hexagons can be observed with such large Pr [21]. For small Biot numbers, Fig. 3(a) shows that squares are never predicted. It can be shown that squares cannot appear for $\mathrm{Bi}<0.28$, for any Prandtl number larger than 1 (see below). For larger Biot numbers [Figs. 3(b) and 3(c)] a critical $\alpha_{c}$ appears that separates regions where rolls or squares are stable. Further remarks are in order about these two pictures. First, one notices that for $\alpha<\alpha_{c}$ the region $H^{+}$where only hexagons can be observed is much smaller than on the right-hand side of $\alpha_{c}$. Second, it is seen that the transition to pure square convection (area $S$ ) occurs for a very large distance from threshold when $\mathrm{Bi}=1$ while a new line allows a transition nearer to the threshold for small $\alpha$ when $\mathrm{Bi}=2$.

We have also examined convection in fluids with a very small Prandtl number such as mercury, for instance. In par-
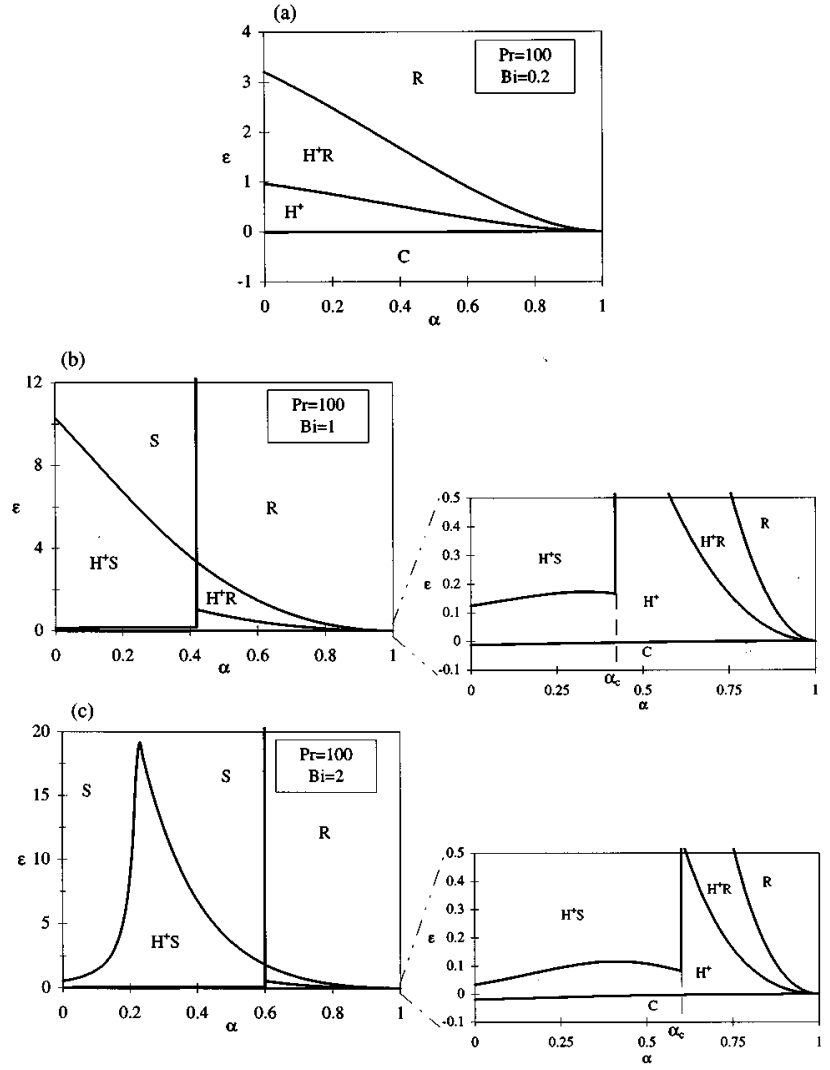

FIG. 3. Stable solutions as functions of $\alpha$ for $\operatorname{Pr}=100$ and for different value of the Biot number. For (b) and (c), closeups of the $\epsilon$ axis are provided on the right.

ticular, we have studied the case $\operatorname{Pr}=0.01$. The corresponding figures will not be given here because these are qualitatively similar to the pictures in Fig. 3. Two main differences should be mentioned, however. First, recall that all stable hexagons are always in this case downflowing hexagons; i.e., the motion is downwards in the center of the convective cells. This is well known and will not be discussed further $[13,21]$. The second difference is that there exists no lower limit for the Biot number under which square cells are never observed. In fact, the behavior described by Fig. 3(a) is not observed for small Prandtl number fluids. The typical situations are in this case either Fig. 3(b) or Fig. 3(c), with only quantitative differences (the different transition lines are displaced in the picture).

A summary of the results when the Biot and Prandtl numbers are varied is provided in Fig. 4. This picture represents the critical $\alpha_{c}$ versus the Prandtl number, for several values of the Biot number. The region above each curve corresponds to stable rolls while stable square convective cells are observed under a curve. For small Pr, squares are always possible, at any value of Biot number larger than or equal to 0 . Moreover, it is noticed that $\alpha_{c}$ decreases with $\mathrm{Bi}$. On the other hand, for large Prandtl numbers, the curve $\alpha_{c}$ disappears when $\mathrm{Bi}<0.28$, which means that only rolls are stable far from the threshold in this case. In addition, it is seen that the curve $\alpha_{c}$ is more sensitive to $\mathrm{Bi}$ for large Pr than for small Pr. So it is necessary to know precisely the value of the 


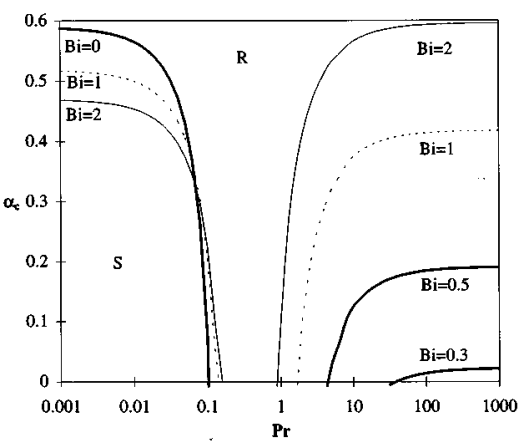

FIG. 4. Critical value $\alpha_{c}$ of parameter $\alpha$ as a function of the Prandtl number and for different Biot numbers. Rolls or squares are stable far from the threshold for $\alpha$ respectively above and below each curve.

Biot number to determine the transition to square convection in this case. Note also that there exists a region in the neighborhood of $\operatorname{Pr}=0.3$ where squares cannot be found, whatever Bi. As a final remark about Fig. 4, let us mention that when the Biot number is increased above $\mathrm{Bi}=2$, the curves $\alpha_{c}$ merge into one another since a saturation effect appears.

It is now interesting to compare our model with the experiments of Nitschke and Thess [22]. In these experiments, the Prandtl number is equal to 100 and the thickness of the fluid is equal to $d=1.55 \mathrm{~mm}$. It is then easy to estimate precisely the value of $\alpha$. From the material properties of the fluid, it is found that $\alpha=0.044$. On the other hand it is well known that the Biot condition used at the top surface is an approximation introduced to avoid solving the complete conservation equations in the gas lying above the fluid layer. In a linear analysis which takes into account the temperature perturbation in the gas, this resolution can be carried out and an accurate value for $\mathrm{Bi}$ can be evaluated. It is easy to show [27] that, in this context,

$$
\mathrm{Bi}=\frac{\lambda_{\text {gas }}}{\lambda_{\mathrm{fl}}} \frac{k}{\tanh \left(k d_{\mathrm{gas}}\right)} .
$$

In this formula, $\lambda_{\text {gas }}$ and $\lambda_{\mathrm{fl}}$ represent the conductivities of the gas and the fluid; $d_{\text {gas }}$ is the dimensionless thickness of the gas layer, while $k$ is the wave number. For the experiments of Nitschke and Thess [22], this "linear Biot number" is equal to 0.44 . Of course this value for $\mathrm{Bi}$ cannot strictly be used in the nonlinear regime for which this parameter is not clearly defined. For these reasons, we give in Fig. 5 the different stable patterns versus the Biot number for the values of $\alpha$ and $\operatorname{Pr}$ corresponding to the experiments of Nitschke and Thess. The shaded area in this picture represents the transition to square patterns as observed experimentally. It is seen that agreement with experimental data is achieved by taking a Biot number equal to about 1.6, which is rather different from the linear value 0.44 . Note finally that, a theoretical work including a complete description of the dynamics of the upper gas layer was performed by Golovin et al.

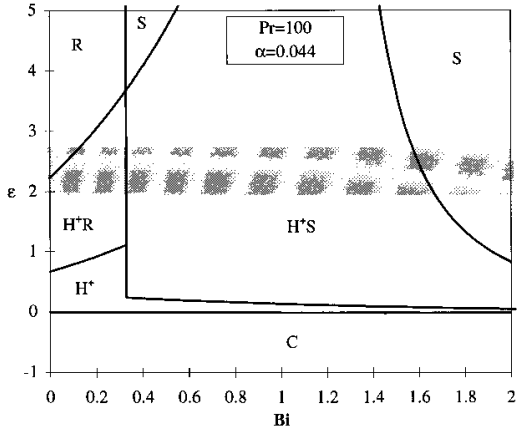

FIG. 5. Stable convective structures as functions of the Biot number for $\alpha=0.044(d=1.55 \mathrm{~mm})$ and $\operatorname{Pr}=100$, which correspond to the experiments of Nitschke and Thess [22]. The shaded area defines the experimental threshold to pure square convection.

[25] for pure Marangoni convection. Their predictions about the transition to square convection are also in good qualitative agreement with the experiments but the results are not completely satisfactory from a quantitative point of view.

\section{CONCLUSION}

The occurrence of square convective cells in a fluid layer heated from below (Bénard-Marangoni problem) has been examined. The mathematical analysis is based on Landau amplitude equations, which were deduced from the general Boussinesq equations. The main conclusions are the following. First, since only rolls are observed for large $\alpha$, it is clear that the appearance of square cells is mainly due to capillary effects and therefore will be observed in thin layers. Typically, squares will never be found for $\alpha$ larger than 0.6 (see Fig. 4), which corresponds to a thickness of the order of 1 $\mathrm{cm}$ for many fluids (silicone oils, mercury, ethanol, glycerol). It is also shown that the Biot number must be larger than 0.28 for squares to be observed in large Prandtl number fluids. When Pr is quite small, squares are possible whatever the value of the Biot number. When the Prandtl number is close to 0.3 , only rolls appear. Note also that the areas where only hexagons are stable are quite smaller when $\alpha<\alpha_{c}$ than for thicker fluid layers. It is also important to stress that qualitative agreement with the experiments of Nitschke and Thess [22] is achieved concerning the transition to square cells. However, the comparison showed the difficulty in defining accurately the Biot number in the nonlinear regime.

\section{ACKNOWLEDGMENTS}

This text presents research results of the Belgian Interuniversity Poles of Attraction (P.A.I. No. 21) initiated by the Belgian State, Prime Minister's Office, Science Policy Programming. The scientific responsibility is assumed by its authors. Partial support from the European Community under Contract No. ERB-CHRX-CT94-0481 is also acknowledged. It is a pleasure to thank Professor J.-C. Legros and his group (Brussels University) for interesting discussions. 
[1] A. Schlüter, D. Lortz, and F. Busse, J. Fluid Mech. 23, 129 (1965).

[2] J. Stuart, J. Fluid Mech. 9, 353 (1960).

[3] L. A. Segel and J. Stuart, J. Fluid Mech. 13, 269 (1962).

[4] L. A. Segel, J. Fluid Mech. 21, 359 (1965).

[5] J. Scanlon and L. A. Segel, J. Fluid Mech. 30, 149 (1967).

[6] J. Bragard and G. Lebon, Europhys. Lett. 21, 831 (1993).

[7] A. Cloot and G. Lebon, J. Fluid Mech. 145, 447 (1984).

[8] P. Cerisier, C. Jamond, J. Pantaloni, and C. Pérez-García, Phys. Fluids 30, 954 (1987).

[9] J. Kraska and R. Sani, Int. J. Heat Mass Transfer 22, 535 (1979).

[10] S. Rosenblat, S. H. Davis, and G. M. Homsy, J. Fluid Mech. 120, 91 (1982); 120, 123 (1982).

[11] W. Eckhaus, Studies in Non-Linear Stability Theory (SpringerVerlag, New York, 1965).

[12] M. C. Cross, Phys. Fluids 23, 1727 (1980); Phys. Rev. A 25, 1065 (1982).

[13] P. C. Dauby, G. Lebon, P. Colinet, and J. C. Legros, Q. J. Mech. Appl. Math. 46, 683 (1993).
[14] M. Bestehorn, Phys. Rev. E 48, 3622 (1993).

[15] A. Thess and M. Bestehorn, Phys. Rev. E 52, 6358 (1995).

[16] P. C. Dauby, Ph.D. thesis, University of Liège, Belgium, 1994.

[17] A. Thess and S. A. Orszag, J. Fluid Mech. 283, 201 (1995).

[18] D. D. Joseph, Stability of Fluid Motions II, Springer Tracts in Natural Philosophy Vol. 28 (Springer-Verlag, Berlin, 1976).

[19] E. L. Koschmieder and S. A. Prahl, J. Fluid Mech. 215, 571 (1990).

[20] E. L. Koschmieder and M. I. Biggerstaff, J. Fluid Mech. 167, 49 (1986).

[21] P. M. Parmentier, V. C. Regnier, G. Lebon, and J. C. Legros, Phys. Rev. E 54, 411 (1996).

[22] K. Nitschke and A. Thess, Phys. Rev. E 52, R5772 (1995).

[23] M. Bestehorn, Phys. Rev. Lett. 76, 46 (1996).

[24] J. Bragard (private communication).

[25] A. A. Golovin, A. A. Nepomnyashchy, and L. M. Pismen (unpublished).

[26] C. Kubstrup, H. Herrero, and C. Pérez-García, Phys. Rev. E 54, 1560 (1996).

[27] C. Normand and Y. Pomeau, Rev. Mod. Phys. 49, 581 (1977). 